\title{
Early Neurologic Deterioration after Stroke Depends on Vascular Territory and Stroke Etiology
}

\author{
James E. Siegler, Alyana Samai, Eleanor Semmes, Sheryl Martin-Schild \\ Tulane Medical Center, New Orleans, LA, USA
}

Background and Purpose Early neurologic deterioration (END) occurs in up to one-third of patients with ischemic stroke and is associated with poor outcomes. The purpose of the present study was to determine which stroke etiologies and vascular distributions pose a greater threat of END in stroke patients.

Methods Using a single-center registry of prospectively maintained clinical data, adult ischemic stroke patients admitted (July 2008 to June 2014) within 48 hours of symptom onset were evaluated according to stroke etiology and vascular distribution using diffusion-weighted MRI. Major stroke etiologies were divided into cardioembolic, large vessel, small vessel, other, unknown source, and multiple possible etiologies. END was defined as a worsening of 2 or more points on the National Institutes of Health Stroke Scale during a 24-hour period of hospitalization. Crude and backward stepwise regression models were generated to associate stroke etiology and vascular distribution with END.

Results Of the included 961 patients (median age 65 years, 47\% female, 72\% non-White), 323 (34\%) experienced END. Strokes involving the internal carotid artery (ICA) were associated with a threefold higher odds of END in stepwise regression models (OR 3.0, 95\% Cl 1.4-6.6, $P=0.006$ ). Among stroke etiologies, those with unclear mechanisms had the lowest odds of END in the fully adjusted model (OR 0.6, 95\% $\mathrm{Cl} 0.4-1.0, P=0.029$ ).

Conclusions In our single-center cohort of patients, ICA infarctions were independently associated with END whereas strokes of unknown etiology were least often associated with END. Larger cohorts are necessary to determine which steps, if any, can be taken to prevent END in these vulnerable populations.

Keywords Diffusion-weighted imaging; Etiology; Ischemic stroke; Early neurologic deterioration; Outcomes research; Risk factors

\section{Introduction}

Early neurologic deterioration (END) occurs in up to one-third of patients with ischemic stroke during the acute hospitalization and is associated with neurologic and functional decline. Investigations have identified risk factors for this process, which include initial stroke severity, ${ }_{1}^{2}$ brainstem infarction, ${ }_{1}^{3}$ significant changes in blood pressure, ${ }^{4}$ diabetes mellitus, ${ }^{3}$ and atrial fibrilla-
Correspondence: James E. Siegler Tulane Medical Center, 1440 Canal Street, TB-52, Suite 1000, New Orleans, LA, USA

Tel: +1-501-912-4392

Fax: +1-504-988-6263

E-mail: siegler.james@gmail.com

Received: January 31, 2016

Revised: May 8, 2016

Accepted: May 9, 2016

The authors have no financial conflicts of interest. 


\section{Methods}

\section{Patient population}

A single-center retrospective investigation of consecutively admitted patients with acute ischemic stroke (July 2008 to June 2014) was conducted using a prospectively maintained clinical registry, as previously described. ${ }^{8}$ Patients 18 years of age or older with acute infarction confirmed on diffusion-weighted magnetic resonance imaging (DMI) were included in the analysis. Patients with an unknown time last seen normal or patients who presented to the emergency department greater than 48 hours after time last seen normal were excluded. Pertinent medical history were documented in the registry if reported by the patient during the initial encounter, or if the information were available via record review. Vascular territories of cerebral infarctions were confirmed using DMI and intracranial vascular imaging (magnetic resonance angiography or computed tomographic angiography) where appropriate. Cerebral infarctions involving multiple arterial distributions were determined by board-certified vascular neurologists according to previously published cerebral arterial maps. ${ }^{9}$ For the purposes of this investigation, only territorial infarctions involving the following arterial distributions were compared: internal carotid artery (ICA), middle cerebral artery (MCA), MCA subcortical branches (MCA perforators), A1 or A2 segments of the anterior cerebral artery, posterior cerebral artery (PCA), subcortical PCA perforators, anterior inferior cerebellar artery, superior cerebellar artery, posterior inferior cerebellar artery, and basilar artery (including its perforators). MCA lenticulostriate and other interior MCA perforating vessels were included in the "MCA perforator" category, as part of our prospective registry's data dictionary, whereas large cortical branches were included in the "MCA" division. PCA thalamoperforators, posterior choroidal arteries and other subcortical PCA branches were included in the "PCA" perforator category, whereas large cortical branches were included in the "PCA" division. Territorial infarctions sustained by fewer than $2 \%$ of the cohort (e.g., anterior choroidal arteries) were not assessed for odds of END given the low incidence and insufficient power to detect statistically significant differences.

At our center, patients ineligible to receive intravenous tissue plasminogen activator (IV tPA) are considered for loading doses of clopidogrel (300-375 mg orally) in addition to standard pharmacologic management for acute ischemic stroke (e.g., aspirin $325 \mathrm{mg}$ and high dose statin therapy unless contraindicated), which we have observed in our population to be safe. ${ }^{10}$

\section{Definitions}

Stroke etiology was determined by a board-certified vascular neurologist according to the Trial of Org 10172 in Acute Stroke Treatment (TOAST)." Major stroke etiologies included cardioembolic source, large vessel source, small vessel source, other source, unknown source, and multiple possible sources, as previously described in our cohort. ${ }^{12}$ For example, strokes due to "other" sources include etiologies such as hypercoagulable state $(n=29)$, cervical artery dissection $(n=16)$, vasculitis $(n=7)$, etc. Patients classified as having multiple possible etiologies include those with combinations of 2 or more potential etiologies such as concomitant atrial fibrillation and hemodynamically significant carotid stenosis-which is the most common etiologic combination we have observed. ${ }^{12}$ National Institutes of Health Stroke Scale (NIHSS) scores are assessed daily at our center by board-certified vascular neurologists in order to evaluate and quantitate our primary outcome measure, END. ${ }^{13}$ In the present investigation, we have defined END as an increase in 2 or more points on the NIHSS (due to any cause) during a 24-hour period during hospitalization. ${ }^{14} \mathrm{~A}$ decline in 2 or more points followed by recovery during hospitalization was also considered END. Such a 2-point worsening in the NIHSS has also been used to define deterioration after stroke in other cohorts. ${ }^{5,16,17}$ Etiologies of END have been previously standardized at our center and include progressive stroke-which we have defined as extension of ischemic findings on neuroimaging that respect the vascular distribution of the initial cerebral infarction-and hemorrhagic transformation which requires follow up neuroimaging to confirm. ${ }^{17}$ Progressive stroke is distinct from END due to edema on the basis of follow-up neuroimaging findings without evidence of tissue swelling despite evolution of symptomatology. Additional etiologies for END include metabolic derangements, seizure, infection, etc., as previously described (Supplementary Table 1). ${ }^{7}$

\section{Statistics}

Categorical data were presented as frequencies and compared using Chi-square, factorial logistic regression, or Fisher's exact test, where appropriate. Continuous data (including END severity) were reported as median with interquartile range (IQR) and compared using Kruskal-Wallis equality-of-populations rank test. Crude logistic regression and logistic regression adjusted for age and baseline NIHSS were used to assess the relationship between stroke etiology (and vascular territory) and END. All variables with a $P$ value of 0.2 and below (age, sex, race, admission NIHSS, admission serum glucose, tobacco use, coronary artery disease history, and thrombolytic treatment) were entered into a backward stepwise regression model to associate stroke etiology or vascular territory with END. In a post hoc analysis, crude and adjusted multivariate logistic regression adjusted for age, sex, stroke severity (admission NIHSS), race, admission serum glucose, 


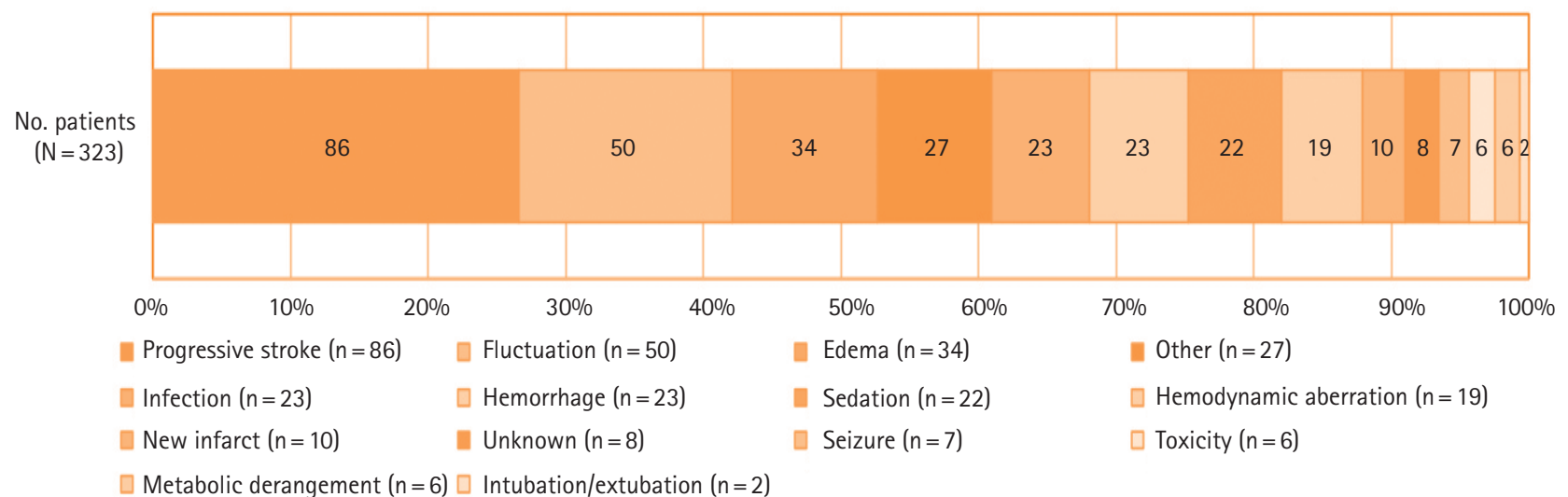

Figure 1. Etiologies of early neurologic deterioration in the cohort. Number within the colored bars represents the number of patients in the cohort who experienced the respective etiology of early neurologic deterioration.

tobacco use, coronary artery disease history, thrombolytic treatment, cryptogenic stroke mechanism, and ICA infarction were performed to identify other independent predictors of END. STATA version 14.0 (College Station, TX, USA) was used for all analyses. $P$ values $<0.05$ were considered statistically significant.

This study, and the clinical registry on which it was founded, were independently approved by our institutional review board.

\section{Results}

Of the 1,387 patients with DWI-confirmed acute ischemic stroke screened in the registry, 961 (69.3\%) met inclusion criteria. The remaining 426 patients were excluded due to presentation $>48$ hours after time last seen normal or unknown time last seen normal. One-hundred eleven patients (11.6\%) had strokes involving more than one arterial distribution and were excluded in subanalyses of vascular territories (Supplementary Table 2). Median time from last seen normal to emergency department arrival was 5 hours 47 minutes (IQR 1 hour 17 minutes-15 hours 39 minutes). Median age was 65 years (IQR 56$75), 47 \%$ were female, and $72 \%$ were non-White (69\% Black). Median length of hospital stay was 5 days (IQR 3-9). Three-hundred twenty three patients (34\%) experienced END with 86 events due to stroke progression (9\% of total cohort, $27 \%$ of END) and 23 due to hemorrhagic transformation (2\% of total cohort, $7 \%$ of END; Figure 1). Compared to patients without END, those with END were older (median age 68 vs. 63 years, $P<0.001$ ), had higher median admission NIHSS (11 vs. $4, P<$ 0.001 ) and serum glucose levels (125 vs. $118 \mathrm{mg} / \mathrm{dL}, P=0.015$ ), and were more likely to have pre-existing coronary artery disease (25.2\% vs. $18.1 \%, P=0.010$ ), but less frequently were active tobacco users (27.7\% vs. $34.9 \%, P=0.026$; Table 1$)$. Patients who received IV tPA were no more likely to experience END than pa- tients who did not receive IV tPA ( $28.5 \%$ vs. $27.8 \%, P=0.828)$.

\section{Stroke etiology}

Twenty-three percent of included patients experienced stroke due to cardioembolic disease, $22 \%$ due to large vessel disease, $22 \%$ due to small vessel disease, $12 \%$ had an alternative etiology diagnosed ("Other"), 16\% had an unknown etiology, and 6\% were diagnosed as having multiple etiologies possible.

When analyzed according to stroke etiology, significant differences in demographic information were observed (Supplementary Table 3). Compared to other etiologies, individuals with cardioembolic stroke were older (71 [IQR 62-81]) and those with "Other" etiologies were younger (58 [IQR 47-67]). Fewer women experienced stroke due to cardioembolic, large vessel, and small vessel disease than the remaining etiologies $(P=0.008)$. Using patients with small vessel disease as a referent, IV tPA was more frequently administered to patients with cardioembolic etiologies, large vessel etiologies, and strokes of unknown etiologies, and this difference remained after adjusting for admission NIHSS and age (odds ratio $[\mathrm{OR}] 2.8,95 \%$ confidence interval [Cl]: 1.74.6, $P<0.001$; OR 1.8, 95\% Cl: 1.1-3.1, $P=0.021$; and OR 3.4, $95 \% \mathrm{Cl}: 2.0-5.7, P<0.001$, respectively).

\section{END}

Among etiologies, END was most frequently observed in patients with "Other" sources of stroke (46.0\%), followed by patients with cardioembolic or large vessel disease (38.5\% for each), and least frequently observed in patients with no known cause $(24.0 \%)$ or strokes due to small vessel disease $(24.8 \%)$ $(P<0.001$; Supplementary Table 2$)$. Patients with "Other" etiologies were at nearly twofold greater odds of END when compared to the remaining stroke population, which persisted in the backward stepwise regression model (OR 1.7, 95\% Cl: 1.1-2.7, 
Table 1. Demographic information by early neurologic deterioration (END)

\begin{tabular}{|c|c|c|c|c|c|c|}
\hline & \multirow[b]{2}{*}{ No END $(n=624)$} & \multirow[b]{2}{*}{$\operatorname{END}(n=323)$} & \multirow[b]{2}{*}{$P$ value } & \multicolumn{3}{|c|}{ Adjusted multivariate logistic regression* } \\
\hline & & & & Odds ratio & $\begin{array}{l}\text { 95\% confidence } \\
\text { interval }\end{array}$ & $P$ value \\
\hline Age, median (interquartile range [IOR]) & $63(55-74)$ & $68(59-78)$ & $<0.001$ & 1.02 & $1.01-1.03$ & 0.004 \\
\hline Sex $\%$ female & $44.8 \%$ & $49.2 \%$ & 0.194 & 0.99 & $0.73-1.34$ & 0.957 \\
\hline Race, \% non-White & $72.0 \%$ & $71.7 \%$ & 0.922 & & & \\
\hline \multicolumn{7}{|l|}{ Past medical history, \% } \\
\hline Hypertension & $79.4 \%$ & $78.6 \%$ & 0.774 & & & \\
\hline Diabetes & $33.6 \%$ & $36.8 \%$ & 0.331 & & & \\
\hline Stroke & $38.4 \%$ & $39.0 \%$ & 0.865 & & & \\
\hline Dyslipidemia & $42.7 \%$ & $41.5 \%$ & 0.727 & & & \\
\hline Active tobacco user & $34.9 \%$ & $27.7 \%$ & 0.026 & 0.87 & $0.63-1.21$ & 0.410 \\
\hline Coronary artery disease & $18.1 \%$ & $25.2 \%$ & 0.010 & 1.42 & $1.00-2.03$ & 0.049 \\
\hline Baseline NIHSS, median (IOR) & $4(2-9)$ & $11(4-18)$ & $<0.001$ & 1.08 & $1.06-1.10$ & $<0.001$ \\
\hline TOAST, \% & & & $<0.001$ & & & \\
\hline Cardioembolic & $21.8 \%$ & $26.3 \%$ & & & & \\
\hline Large vessel & $21.0 \%$ & $25.4 \%$ & & & & \\
\hline Small vessel & $24.8 \%$ & $15.8 \%$ & & & & \\
\hline Other & $9.6 \%$ & $15.8 \%$ & & & & \\
\hline No known cause & $17.8 \%$ & $10.8 \%$ & & 0.62 & $0.40-0.96$ & 0.032 \\
\hline Multiple possible causes & $5.0 \%$ & $5.9 \%$ & & & & \\
\hline Admission glucose, mg/dL, median (IQR) & $118(100-150)$ & $125(103-165)$ & 0.015 & 1.00 & $1.00-1.00$ & 0.511 \\
\hline $\begin{array}{l}\text { Intravenous tissue plasminogen activator } \\
\text { received, \% }\end{array}$ & $27.8 \%$ & $28.5 \%$ & 0.828 & 0.72 & $0.51-1.02$ & 0.065 \\
\hline
\end{tabular}

*Multivariate logistic regression adjusted for age, sex, admission NIHSS, race, admission serum glucose, tobacco use, coronary artery disease history, cryptogenic stroke mechanism, internal carotid artery infarction, and intravenous thrombolytic therapy.

$P=0.020$; Table 2). Conversely, patients with unknown stroke etiologies and those with small vessel disease had the lowest odds of END, and this reduction persisted in the stepwise regression model for patients with an unknown stroke etiology (OR 0.6, 95\% Cl: 0.4-1.0, $P=0.029$; Table 2).

Using small vessel disease as a referent (having the lowest odds of END), patients with strokes due to cardioembolic sources, large vessel disease, or strokes due to "Other" causes were at greater odds of END (OR 1.9, 95\% Cl: 1.3-2.9, $P=0.003$; OR 1.9, 95\% Cl: 1.3-2.9, $P=0.003$; and OR 2.6, 95\% Cl: 1.6-4.2, $P<$ 0.001 , respectively). In this referential model, after adjusting for confounders including age, NIHSS at baseline, and IV tPA use, only strokes of "Other" causes were independently associated with a greater odds of END (OR 2.0, 95\% Cl: 1.2-3.4, $P=0.011$ ).

Among vascular distributions, ICA occlusions were strongly associated with END, and this vascular distribution conferred the highest odds of END among all vascular territories in the stepwise regression model (OR 3.0, 95\% Cl: 1.4-6.6, $P=0.006$; Table 2). There was no significant relationship between basilar occlusions (including basilar perforating vessels), or MCA occlusions and END (Table 2). MCA perforators also failed to correlate with END. When analyzed uniquely, complete basilar occlusion (excluding perforators) was also unrelated to END in univariate regression (OR 2.2, 95\% Cl: 0.8-6.2, $P=0.123$ ).
Progressive stroke, which our center defines as an extension of ischemic injury of the affected vascular territory, was identified as the etiology of END for $26.6 \%$ of all END. When compared across stroke etiologies, stroke progression was the most common cause of END in large vessel strokes (40.2\%), and the least common cause of END in "Other" sources of stroke (13.7\%, $P<0.001$ by Fisher's exact test). Due to the low number of other identifiable END etiologies, there was no significant association between stroke etiology (or vascular territory) and END etiology (data not shown).

In the post hoc analysis to identify other factors independently associated with END, older age, greater baseline stroke severity (according to admission NIHSS), and history of coronary artery disease were strongly associated with END (Table 1). Infarctions of the ICA distribution also remained significantly associated with END in this model (OR 2.5, 95\% Cl: 1.2-5.2, P=0.013). Conversely, strokes attributed to unknown etiology remained inversely related to END (OR 0.6, 95\% Cl: 0.4-1.0, $P=0.032$; Table 1).

\section{Discussion}

Our study has two main findings. First, patients with infarcts of unknown etiology had a lower likelihood of END than infarctions due to known stroke etiologies. Second, infarctions of the 
Table 2. Odds of early neurologic deterioration (END) according to stroke etiology and vascular territory.

\begin{tabular}{|c|c|c|c|c|c|c|}
\hline Stroke etiology & $\begin{array}{l}\text { Unadjusted odds of END } \\
\text { (95\% confidence interval } \\
\text { [Cl]) }\end{array}$ & $P$ value & $\begin{array}{l}\text { Adjusted odds of END } \\
(95 \% \mathrm{Cl})^{*}\end{array}$ & $P$ value & $\begin{array}{l}\text { Stepwise regression, odds } \\
\text { ratio (OR) }(95 \% \mathrm{Cl})^{+}\end{array}$ & $P$ value \\
\hline Cardioembolic source $(n=223)$ & $1.3(0.9-1.8)$ & 0.121 & $0.9(0.7-1.3)$ & 0.697 & Not significant (NS) & \\
\hline Large vessel source $(n=214)$ & $1.3(0.9-1.8)$ & 0.127 & $1.2(0.9-1.7)$ & 0.271 & NS & \\
\hline Small vessel source $(n=209)$ & $0.6(0.4-0.8)$ & 0.001 & $0.9(0.6-1.3)$ & 0.446 & NS & \\
\hline Other source $(n=113)$ & $1.8(1.2-2.6)$ & 0.006 & $1.9(1.2-2.9)$ & 0.005 & $1.7(1.1-2.7)$ & 0.020 \\
\hline Unknown source $(n=149)$ & $0.6(0.4-0.8)$ & 0.004 & $0.6(0.4-0.9)$ & 0.012 & $0.6(0.4-1.0)$ & 0.029 \\
\hline Multiple etiologies possible $(n=53)$ & $1.2(0.7-2.2)$ & 0.554 & $1.0(0.6-2.0)$ & 0.908 & NS & \\
\hline Vascular territory & $\begin{array}{l}\text { Unadjusted odds of END } \\
\qquad(95 \% \mathrm{Cl})\end{array}$ & $P$ value & $\begin{array}{l}\text { Adjusted odds of END } \\
(95 \% \mathrm{Cl})^{*}\end{array}$ & $P$ value & $\begin{array}{l}\text { Stepwise regression, OR } \\
(95 \% \mathrm{Cl})^{+}\end{array}$ & $P$ value \\
\hline Internal carotid artery $(n=34)$ & $4.6(2.2-9.6)$ & $<0.001$ & $3.0(1.4-6.6)$ & 0.006 & $3.0(1.3-6.8)$ & 0.008 \\
\hline $\begin{array}{l}\text { Middle cerebral artery (MCA) } \\
\quad(n=416)\end{array}$ & $1.0(0.7-1.3)$ & 0.920 & $0.7(0.5-1.0)$ & 0.055 & $0.8(0.6-1.0)$ & 0.128 \\
\hline MCA perforators $(n=154)$ & $0.6(0.4-0.9)$ & 0.007 & $0.8(0.5-1.2)$ & 0.347 & NS & \\
\hline Anterior cerebral artery $(n=25)$ & $1.0(0.4-2.3)$ & 0.947 & $1.1(0.4-2.7)$ & 0.892 & NS & \\
\hline Basilar $(n=54)$ & $1.1(0.6-2.0)$ & 0.677 & $1.5(0.9-2.4)$ & 0.164 & NS & \\
\hline $\begin{array}{l}\text { Posterior inferior cerebellar artery } \\
(n=10)\end{array}$ & $0.5(0.1-2.4)$ & 0.400 & $1.4(0.8-2.7)$ & 0.190 & NS & \\
\hline $\begin{array}{l}\text { Posterior cerebral artery (PCA) } \\
(n=41)\end{array}$ & $1.1(0.6-2.1)$ & 0.830 & $1.2(0.6-2.4)$ & 0.646 & NS & \\
\hline PCA perforators $(n=24)$ & $0.7(0.3-1.7)$ & 0.422 & $0.8(0.3-2.0)$ & 0.604 & NS & \\
\hline
\end{tabular}

Odds provided here for probability of experiencing END for a patient who experienced a stroke due to a given etiology when compared to all remaining etiologies, or stroke involving the respective vascular distribution when compared to all remaining vascular territories. Strokes involving multiple vascular territories $(\mathrm{N}=111$ patients) were excluded in this subgroup analysis.

${ }^{*}$ Adjusted regression for age and baseline National Institutes of Health Stroke Scale (NIHSS) only; ${ }^{+}$Backward stepwise regression was performed using all variables significant to $P<0.2$ (age, sex, race, admission NIHSS, admission serum glucose, tobacco use, coronary artery disease history, and thrombolytic treatment).

ICA were highly associated with END, even after adjustment for confounding variables such as age and stroke severity.

\section{END by stroke etiology}

Among stroke etiologies, small vessel infarctions also carried a benign course and low likelihood of END in this cohort (24.8\% of cases experienced END), as in previous investigations. ${ }^{2,7}$ However, after adjustment for known confounders in the stepwise regression model (including the less severe baseline NIHSS), small vessel disease had no effect on the odds of END or END due to stroke progression. This may be attributed to the fact that stroke providers at our center frequently administer oral loading doses of clopidogrel (300-375 mg) with or without aspirin for patients with lacunar infarcts who are not eligible for IV tPA because this antiplatelet combination appears to confer no greater risk of intracranial hemorrhage ${ }^{10,18}$ and may even reduce the risk of END. ${ }^{19}$

The observed neutral risk of END in small vessel disease confirms findings from one previous study. In that single-center cohort of over 3,000 stroke patients, the investigators observed a higher incidence of END among patients with infarctions due to large vessel disease as opposed to lacunar and cardioembolic infarctions. However, compared to the present study, Yamamoto and colleagues defined END qualitatively according to witnessed clinical change by physicians and nursing staff. These investigators also did not distinguish between the varying etiologies of END, as we have described here. Furthermore, this study reported a higher proportion of younger patients with clinical deterioration when compared to the elderly. This finding contradicts that of other studies including those from our cohort, ${ }^{6,20}$ and remains incompletely elucidated.

Cardioembolic strokes were typically the most severe and occurred in the older patient group, consistent with prior data. ${ }^{21}$ Comparatively, these strokes were more frequently treated with IV tPA, and carried a moderately increased odds of END (38.5\%). In these patients END was infrequently attributed to stroke progression (16.5\% of END). In adjusted models, there appeared to be no greater odds of END in cardioembolic strokes, as in one study by Yamamoto et al. ${ }^{7}$ Compared to patients with small vessel infarctions, however, the odds of END in cardioembolic disease became twice as great. The higher risk of END attributed to cardioembolic strokes appears to be attenuated by the greater severity, older age, and IV tPA treatment of these patients compared to those with strokes due to small vessel disease.

Strokes due to large vessel disease also carried a moderately increased odds of END among all stroke etiologies, as in the study by Yamamoto and colleagues.' Of the etiologies of END, 
stroke progression was the most common cause of worsening, accounting for $40.2 \%$ of all END, which can be explained by the proposed mechanisms of clot propagation and vasogenic ede$\mathrm{ma}^{22,23}$ As in the case for cardioembolic strokes, strokes due to large vessel disease were at nearly twice the odds of END compared to strokes due to small vessel disease, however this significant difference attenuates when stroke severity, age, and IV IPA use are incorporated into an adjusted model.

Among the heterogeneous etiologies of cerebral infarction, strokes attributed to "Other" causes carried the highest risk of END during hospitalization in our cohort and, in backward stepwise regression, were still associated with a nearly two-fold greater odds of END. In contrast, strokes with unclear mechanisms were associated with the lowest odds of END in fully adjusted models.

However, given the heterogeneous causes of stroke in these categories and the relatively low number of patients with "Other" strokes, it is difficult to derive conclusions from these findings. These results should be interpreted as hypothesis generating.

\section{END by vascular distribution}

Among the vascular distributions, ICA infarcts were strongly and independently associated with END. This observation may be attributed to the greater amount of real estate afforded to strength testing (a largely anterior circulation process) and language function on the NIHSS, ${ }^{13,24}$ but it may also reflect the greater variety of etiologies, which may impact the deterioration process. Anterior supratentorial strokes are more frequently associated with edema formation, midline shift and hemorrhage which can promote END. ${ }^{25}$ In contrast to previous studies, ${ }^{3,26}$ we found no significant risk of END among posterior circulation infarctions, infarctions of the basilar artery, or anterior choroidal artery infarctions. Although END appeared unrelated to basilar occlusions in our cohort, which typically portend a devastating prognosis, this may be explained by the fact that all basilar strokes (including strokes of small perforating vessels) were grouped together. Small straight and perforating vessels originating from the basilar may have produced less severe, or less clinically evolving, ${ }_{1}^{27}$ deficits compared to deficits sustained by evolving strokes of the entire posterior circulation in the setting of total basilar occlusion. Therefore, some of the smaller strokes due to basilar straight and circumferential perforator occlusions may have driven the lack of association with END. When analyzed separately from basilar perforators, total basilar occlusions conferred a twofold higher odds of END, but this was not statistically significant.

\section{Limitations}

This investigation was limited largely by its retrospective, cross-sectional design and single-center cohort. While intracranial vessel imaging would have been a preferred modality for precise anatomic localization of infarction, diffusion-weighted magnetic resonance imaging was felt by the investigators to optimally and reliably assess arterial distribution as in previous studies. ${ }^{6.26}$ It may even be superior to computed tomographic or magnetic resonance angiography in detecting small vessel occlusions, particularly after recanalization. Due to low numbers of strokes within the distributions of the anterior and posterior choroidal arteries $(0.5 \%$ and $0.2 \%$, respectively), anterior cerebral artery perforators $(0.3 \%)$, vertebral artery and its perforators $(0.9 \%$ and $0.8 \%$, respectively), and other small vessels, these distributions were excluded from analyses. Although terminal vessels of the MCA trunk were included in the analysis, we made no distinction between interior perforators (e.g., lenticulostriate vessels) and distal subcortical branches (e.g., interior perforators of the M3 and M4 divisions). It is assumed that more proximal MCA occlusions put patients at greater risk of END, and this subject has already been explored in detail, ${ }_{1}^{28,29}$ so we do not believe this limitation to significantly detract from our other findings. The risk of END is expected to be greater for more proximal or medially deviating branches of the MCA and anterior cerebral artery trunks, as in the case of anterior choroidal infarctions, ${ }^{26}$ when compared to the more distal or externally deviating branches. This deserves further scrutiny in follow-up investigations. Furthermore, the inclusion of lesion size was not evaluated in the present study as it is not a variable collected in our prospective registry. That being said, it has been known since the design of the NIHSS that infarct size correlates with the NIHSS, ${ }^{30}$ however the added factor of stroke location to volume size more reliably predicts NIHSS, ${ }^{31}$ a finding that represented the focus of this investigation.

\section{Summary}

Despite these limitations, our data include a robust collection of daily NIHSS scores for patients admitted with acute stroke to our center with extensive documentation as to the etiology of END, in contrast to prior studies. 'This has permitted us to quantitate small changes in the neurologic exam of stroke patients throughout a given hospitalization, and to identify episodes of clinical deterioration. Because subtle changes may not be so easily recognized (or reliably quantified) on routine neurologic examination, use of the NIHSS may prove to be an effective tool in capturing events of END. ${ }^{13}$ Even a 2-point worsening in NIHSS has shown to be associated with adverse outcomes, including a 
3-fold higher rate of in-hospital mortality, as our center has demonstrated. ${ }^{14}$ Although it is not part of the current guidelines for acute stroke management, ${ }_{1}^{32}$ we recommend at least daily assessment of stroke patients using the NIHSS, in particular for those patients at high risk of END. ${ }^{13}$

The present study adds to the impressive body of literature on END by identifying which stroke etiologies and vascular distributions should alert providers to be more attentive to any small clinical change. In particular, ICA and PCA infarctions appear to carry the greatest risk of END. In contrast, strokes due to intracranial atherosclerosis pose a significantly lower risk of early deterioration. The early identification of these known, at-risk patients may permit the clinician to more readily recognize and rapidly intervene upon potentially reversible causes of deterioration after stroke. ${ }^{17}$

\section{Acknowledgments}

The authors would like to acknowledge the statistical support of Dominique J. Monlezun.

\section{References}

1. Siegler JE, Martin-Schild S. Early Neurological Deterioration (END) after stroke: the END depends on the definition. Int J Stroke 2011;6:211-212.

2. Adams HP Jr, Davis PH, Leira EC, Chang KC, Bendixen BH, Clarke WR, et al. Baseline NIH Stroke Scale score strongly predicts outcome after stroke: a report of the Trial of Org 10172 in Acute Stroke Treatment (TOAST). Neurology 1999;53:126131.

3. Weimar C, Mieck T, Buchthal J, Ehrenfeld CE, Schmid E, Diener $\mathrm{HC}$; German Stroke Study Collaboration. Neurologic worsening during the acute phase of ischemic stroke. Arch Neurol 2005; 62:393-397.

4. Castillo J, Leira R, Garcia MM, Serena J, Blanco M, Dávalos A. Blood pressure decrease during the acute phase of ischemic stroke is associated with brain injury and poor stroke outcome. Stroke 2004;35:520-526.

5. Kwan J, Hand P. Early neurological deterioration in acute stroke: clinical characteristics and impact on outcome. $O / M$ 2006;99:625-633.

6. Siegler JE, Boehme AK, Kumar AD, Gillette MA, Albright KC, Beasley TM, et al. Identification of modifiable and nonmodifiable risk factors for neurologic deterioration after acute ischemic stroke. J Stroke Cerebrovasc Dis 2013;22:e207-e213.

7. Yamamoto H, Bogousslavsky J, van Melle G. Different predictors of neurological worsening in different causes of stroke.
Arch Neurol 1998;55:481-486.

8. Siegler JE, Boehme AK, Dorsey AM, Monlezun DJ, George AJ, Shaban $A$, et al. A comprehensive stroke center patient registry: advantages, limitations, and lessons learned. Med Students Res J 2013;2:21-29.

9. Tatu L, Moulin T, Vuillier F, Bogousslavsky J. Arterial territories of the human brain. Front Neurol Neurosci 2012;30:99-110.

10. Leung LY, Albright KC, Boehme AK, Tarsia J, Shah KR, Siegler

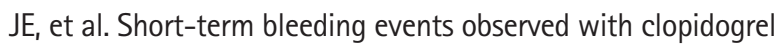
loading in acute ischemic stroke patients. J Stroke Cerebrovasc Dis 2013;22:1184-1189.

11. Adams HP Jr, Bendixen BH, Kappelle U, Biller J, Love BB, Gordon $\mathrm{DL}$, et al. Classification of subtype of acute ischemic stroke. Definitions for use in a multicenter clinical trial. TOAST. Trial of Org 10172 in Acute Stroke Treatment. Stroke 1993;24:35-41.

12. Scullen TA, Monlezun DJ, Siegler JE, George AJ, Schwickrath $M, E l$ Khoury R, et al. Cryptogenic stroke: clinical consideration of a heterogeneous ischemic subtype. J Stroke Cerebrovasc Dis 2015;24:993-999.

13. Siegler JE, Martin-Schild S. Daily National Institutes of Health Stroke Scale examinations at stroke centers: why not do them? Int J Stroke 2015;10:140-142.

14. Siegler JE, Boehme AK, Kumar AD, Gillette MA, Albright KC, Martin-Schild $S$. What change in the national institutes of health stroke scale should define neurologic deterioration in acute ischemic stroke? J Stroke Cerebrovasc Dis 2013;22:675682.

15. Grotta JC, Welch KM, Fagan SC, Lu M, Frankel MR, Brott T, et al. Clinical deterioration following improvement in the NINDS rt-PA Stroke Trial. Stroke 2001;32:661-668.

16. Toyoda K, Fujimoto S, Kamouchi M, lida M, Okada Y. Acute blood pressure levels and neurological deterioration in different subtypes of ischemic stroke. Stroke 2009;40:2585-2588.

17. Siegler JE, Boehme AK, Albright KC, George AJ, Monlezun DJ, Beasley TM, et al. A proposal for the classification of etiologies of neurologic deterioration after acute ischemic stroke. JStroke Cerebrovasc Dis 2013;22:e549-e556.

18. Wang Y, Pan Y, Zhao X, Li H, Wang D, Johnston SC, et al. Clopidogrel with Aspirin in Acute Minor Stroke or Transient Ischemic Attack (CHANCE) Trial: one-year outcomes. Circulation 2015;132:40-46.

19. He F, Xia C, Zhang JH, Li XQ, Zhou ZH, Li FP, et al. Clopidogrel plus aspirin versus aspirin alone for preventing early neurological deterioration in patients with acute ischemic stroke. $J$ Clin Neurosci 2015;22:83-86.

20. Dávalos $A$, Toni $D$, Iweins $F$, Lesaffre $E$, Bastianello $S$, Castillo J. Neurological deterioration in acute ischemic stroke: potential predictors and associated factors in the European cooperative 
acute stroke study (ECASS) I. Stroke 1999;30:2631-2636.

21. Schulz UG, Rothwell PM. Differences in vascular risk factors between etiological subtypes of ischemic stroke: importance of population-based studies. Stroke 2003;34:2050-2059.

22. Fisher M, Garcia JH. Evolving stroke and the ischemic penumbra. Neurology 1996;47:884-888.

23. Thanvi B, Treadwell S, Robinson T. Early neurological deterioration in acute ischaemic stroke: predictors, mechanisms and management. Postgrad Med J 2008;84:412-417.

24. Kasner SE. Clinical interpretation and use of stroke scales. Lancet Neurol 2006;5:603-612.

25. Bamford J, Sandercock P, Dennis M, Burn J, Warlow C. Classification and natural history of clinically identifiable subtypes of cerebral infarction. Lancet 1991;337:1521-1526.

26. Derflinger S, Fiebach JB, Böttger $S$, Haberl RL, Audebert HJ. The progressive course of neurological symptoms in anterior choroidal artery infarcts. Int J Stroke 2015;10:134-137.

27. Kim JS, Cho KH, Kang DW, Kwon SU, Suh DC. Basilar artery atherosclerotic disease is related to subacute lesion volume increase in pontine base infarction. Acta Neurol Scand 2009;
120:88-93.

28. Kasner SE, Demchuk AM, Berrouschot J, Schmutzhard E, Harms L, Verro $P$, et al. Predictors of fatal brain edema in massive hemispheric ischemic stroke. Stroke 2001;32:2117-2123.

29. Ng YS, Stein J, Ning M, Black-Schaffer RM. Comparison of clinical characteristics and functional outcomes of ischemic stroke in different vascular territories. Stroke 2007;38:23092314.

30. Brott T, Adams HP Jr, Olinger CP, Marler JR, Barsan WG, Biller $J$, et al. Measurements of acute cerebral infarction: a clinical examination scale. Stroke 1989;20:864-870.

31. Menezes NM, Ay $H$, Wang Zhu M, Lopez CJ, Singhal AB, Karonen JO, et al. The real estate factor: quantifying the impact of infarct location on stroke severity. Stroke 2007;38: 194-197.

32. Jauch EC, Saver JL, Adams HP Jr, Bruno A, Connors JJ, Demaerschalk $B M$, et al. Guidelines for the early management of patients with acute ischemic stroke: a guideline for healthcare professionals from the American Heart Association/American Stroke Association. Stroke 2013;44:870-947. 
Supplementary Table 1. Definitions of various etiologies of early neurological deterioration according to our center's predefined registry

\begin{tabular}{|c|c|}
\hline $\begin{array}{l}\text { Etiology of early neurologic } \\
\text { Deterioration (END) }\end{array}$ & Definition \\
\hline \multicolumn{2}{|l|}{ Reversible } \\
\hline Infectious & $\begin{array}{l}\text { END in the presence of a diagnosed infection (e.g., of the urinary or respiratory tracts, cerebral ventricles or meninges, skin or } \\
\text { soft tissue, blood vessels or heart valves)* with identified pathogen on microbiology or consolidation on chest imaging with } \\
\text { clinical symptoms, in the case of pneumonia }\end{array}$ \\
\hline Metabolic derangement & $\begin{array}{l}\text { END in the presence of new laboratory derangement based on our center's laboratory standards (e.g., uremia, hyper/hypocal } \\
\text { cemia, hyper/hypoglycemia, hyper/hyponatremia, or hyperammonemia) }\end{array}$ \\
\hline Hemodynamic & $\begin{array}{l}\text { END in the presence of a significant rise or fall in blood pressure during the } 24 \text { hours prior to documentation of END as de } \\
\text { termined clinically, new-onset arrhythmia as determined via electrocardiography or telemetry, or cardiovascular event (in } \\
\text { cluding myocardial infarction) as determined via electrocardiographic, telemetric, and/or laboratory methods (for instance, } \\
\text { elevated serum troponin I level }\left.\right|^{+} \text {) that may have impaired cerebral perfusion but did not cause a new imaging-confirmed } \\
\text { infarction }\end{array}$ \\
\hline Edema & $\begin{array}{l}\text { END when focal cerebral or cerebellar mass effect was identified on follow-up computed tomographic or magnetic reso } \\
\text { nance imaging in the 24-hour window surrounding the date of END }\end{array}$ \\
\hline Fluctuation & $\begin{array}{l}\text { END in the absence of an identifiable cause should the patient return to prior (day before END) National Institutes of Health } \\
\text { Stroke Scale score within } 24 \text { hours of the episode of decline }\end{array}$ \\
\hline Toxicity & END co-occurrence with the administration of medication with known sedative effects \\
\hline Seizure & $\begin{array}{l}\text { END in the presence of electroencephalogram-confirmed epileptiform activity or periodic rhythm or clinical observation of } \\
\text { seizure-like activity during the 24-hour window surrounding documentation of END }\end{array}$ \\
\hline \multicolumn{2}{|r|}{ 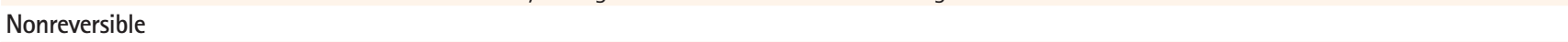 } \\
\hline New stroke & $\begin{array}{l}\text { END in the presence of new ischemic findings on CT or MRI (detected within the 24-hour window surrounding documenta } \\
\text { tion of END) }\end{array}$ \\
\hline Progressive stroke & $\begin{array}{l}\text { END due to extension of ischemic findings as detected on computed tomographic or magnetic resonance imaging by the at } \\
\text { tending physician or staff radiologist within the distribution of the initial vessel occlusion (during the 24-hour window sur } \\
\text { rounding documentation of END), and no clear focal cerebral edema identified on neuroimaging }\end{array}$ \\
\hline Hemorrhagic conversion & $\begin{array}{l}\text { END due to new or progressive hemorrhage of parenchymal hematoma type } 1 \text { or } 2 \text { grade was identified on 24-hour follow- } \\
\text { up imaging study }\end{array}$ \\
\hline Cardiopulmonary arrest & END following cardiopulmonary arrest \\
\hline Unknown etiology & $\begin{array}{l}\text { END without a clear etiologic identification by the attending physician, and none of the aforementioned findings were docu } \\
\text { mented in the progress notes }\end{array}$ \\
\hline
\end{tabular}

*Bacteremia was defined growth of bacterium on culture from a venous blood sample (excluding common contaminants). Urinary tract infection was defined as $>10,000$ colony forming units per millimeter of urine in a clean-catch specimen (excluding contaminants) or suggestive findings on urinalysis. Pneumonia was defined as an infiltrate on chest radiography with appropriate clinical correlates. Other infection types were diagnosed clinically or via laboratory/imaging findings. Clinical symptoms producing END may have occurred hours or days prior to the diagnosis of END due to infection, depending on the manner in which the infection was diagnosed. For example, pneumonia diagnosed via chest radiography in the presence of clinical symptoms may only take one hour for the imaging order to be processed, performed and interpreted, whereas confirmation of bacterial specimen in blood culture may have taken up to 5 days according to our laboratory standards; ${ }^{\dagger}$ Elevated serum troponin I is defined as troponin I level $>0.015 \mathrm{mg} / \mathrm{dL}$, according to our laboratory standards. 
Supplementary Table 2. Arterial distributions of strokes involving more than one vessel territory $(N=87)$

\begin{tabular}{|c|c|c|c|c|c|c|c|}
\hline & $\begin{array}{l}\text { Internal carotid } \\
\text { artery (ICA) }\end{array}$ & $\begin{array}{l}\text { Middle cerebral } \\
\text { artery (MCA) }\end{array}$ & MCA perforator & $\begin{array}{c}\text { Anterior cerebral } \\
\text { artery }\end{array}$ & Basilar & $\begin{array}{l}\text { Posterior inferior } \\
\text { cerebellar artery } b\end{array}$ & $\begin{array}{l}\text { Posterior cere- } \\
\text { bral artery (PCA) }\end{array}$ \\
\hline ICA & - & - & - & - & - & - & - \\
\hline MCA & 0 & - & - & - & - & - & - \\
\hline MCA perforator & 0 & 0 & - & - & - & - & - \\
\hline Anterior cerebral artery & 0 & 0 & 2 & - & - & - & - \\
\hline Basilar & 1 & 0 & 4 & 0 & - & - & - \\
\hline Posterior inferior cerebellar artery & 1 & 11 & 3 & 0 & 0 & - & - \\
\hline PCA & 5 & 27 & 5 & 5 & 0 & 15 & - \\
\hline PCA perforator & 0 & 1 & 5 & 0 & 0 & 2 & 0 \\
\hline
\end{tabular}

Table excludes arterial distributions affecting $<2 \%$ of patients after exclusion of strokes affecting multiple vascular distributions. This left 87 points of overlap. 
Supplementary Table 3. Demographic information by stroke etiology

\begin{tabular}{|c|c|c|c|c|c|c|c|}
\hline & $\begin{array}{l}\text { Cardioembolic } \\
\text { source }(n=223)\end{array}$ & $\begin{array}{l}\text { Large vessel } \\
\text { source }(n=214)\end{array}$ & $\begin{array}{c}\text { Small vessel } \\
\text { source }(n=209)\end{array}$ & $\begin{array}{l}\text { Other source } \\
\quad(n=113)\end{array}$ & $\begin{array}{l}\text { No known source } \\
\quad(n=149)\end{array}$ & $\begin{array}{l}\text { Multiple possible } \\
\text { sources }(n=53)\end{array}$ & $P$ value \\
\hline Age, median (interquartile range [IOR]) & $71(62-81)$ & $65(59-76)$ & $63(56-71)$ & $58(47-67)$ & $61(52-73)$ & $64(51-73)$ & $<0.001$ \\
\hline Sex, \% female & $43.7 \%$ & $42.5 \%$ & $40.7 \%$ & $54.9 \%$ & $57.7 \%$ & $45.3 \%$ & 0.008 \\
\hline Race, \% non-White & $68.6 \%$ & $73.4 \%$ & $76.6 \%$ & $69.6 \%$ & $69.6 \%$ & $75.5 \%$ & 0.451 \\
\hline \multicolumn{8}{|l|}{ Past medical history, \% } \\
\hline Hypertension & $80.5 \%$ & $80.8 \%$ & $82.3 \%$ & $73.9 \%$ & $73.7 \%$ & $76.9 \%$ & 0.273 \\
\hline Diabetes & $33.0 \%$ & $34.4 \%$ & $38.8 \%$ & $32.1 \%$ & $35.8 \%$ & $28.9 \%$ & 0.702 \\
\hline Stroke & $41.0 \%$ & $39.8 \%$ & $35.9 \%$ & $42.3 \%$ & $36.2 \%$ & $36.5 \%$ & 0.792 \\
\hline Dyslipidemia & $43.0 \%$ & $46.5 \%$ & $41.2 \%$ & $41.4 \%$ & $38.9 \%$ & $42.3 \%$ & 0.794 \\
\hline Active tobacco user & $20.1 \%$ & $39.8 \%$ & $43.0 \%$ & $25.0 \%$ & $30.4 \%$ & $33.3 \%$ & $<0.001$ \\
\hline Coronary artery disease & $29.2 \%$ & $19.0 \%$ & $19.1 \%$ & $20.7 \%$ & $12.8 \%$ & $21.6 \%$ & 0.016 \\
\hline $\begin{array}{l}\text { Baseline National Institutes of Health } \\
\text { Stroke Scale, median (IOR) }\end{array}$ & $9(4-17)$ & $7(3-14)$ & $4(2-6)$ & $8(3-19)$ & $5(2-12)$ & $9(3-19)$ & $<0.001$ \\
\hline $\begin{array}{l}\text { Admission glucose, median mg/dL } \\
\text { (IQR) }\end{array}$ & $122(100-151)$ & 119 (101-157) & 116 (98-165) & $122(103-168)$ & $120(103-159)$ & $121(101-141)$ & 0.944 \\
\hline $\begin{array}{l}\text { Intravenous tissue plasminogen } \\
\text { activator received, \% }\end{array}$ & $37.4 \%$ & $27.1 \%$ & $12.9 \%$ & $24.8 \%$ & $38.3 \%$ & $32.7 \%$ & $<0.001$ \\
\hline Early neurologic deterioration (END), \% & $38.5 \%$ & $38.5 \%$ & $24.8 \%$ & $46.0 \%$ & $24.0 \%$ & $38.0 \%$ & $<0.001$ \\
\hline $\begin{array}{l}\text { END from progressive stroke, \% (\% of } \\
\text { END) }\end{array}$ & $6.3 \%(16.5 \%)$ & $15.4 \%(40.2 \%)$ & $7.2 \%(29.4 \%)$ & $6.2 \%(13.7 \%)$ & $7.4 \%(31.4 \%)$ & $11.3 \%(31.6 \%)$ & $\begin{array}{c}0.014 \\
(0.003)\end{array}$ \\
\hline
\end{tabular}

